Citation: Daşdemir, A . "On the Norms of Toeplitz Matrices with the Pell, Pell-Lucas and Modified Pell Numbers". Journal of Engineering Technology and Applied Sciences 1 (2) $2016: 51-57$

\title{
ON THE NORMS OF TOEPLITZ MATRICES WITH THE PELL, PELL-LUCAS AND MODFIED PELL NUMBERS
}

\author{
Ahmet Daşdemir \\ Department of Mathematics, Faculty of Arts and Sciences, Kastamonu University, Kastamonu Turkey \\ ahmetdasdemir37@gmail.com
}

\begin{abstract}
This paper deals with the usual Pell, Pell-Lucas and Modified Pell numbers and Toeplitz matrices. First, the Toeplitz matrices whose entries are the usual Pell, Pell-Lucas and Modified Pell numbers are constructed, and then the Frobenius, row and column norms of such matrices are computed. Furthermore, the upper and lower bounds for the spectral norms of these matrices are obtained.
\end{abstract}

Keywords: Pell Sequence, Pell-Lucas Sequence, Modified Pell Sequence, Toeplitz Matrix, Norm.

\section{Introduction}

In the literature, there exist a lot of integer sequences defined by a recurrence relation. Admittedly, the Fibonacci sequence is one of the most famous and curious integer sequences in mathematics and has been widely studied from both algebraic and combinatorial prospective. It is an inexhaustible source of many interesting identities. The monograph in $[1,2]$ presents the fairly extensive systematic investigation on the subject.

The above-mentioned statements can be said for the Pell, Pell-Lucas and Modified Pell sequences, which is as important as the Fibonacci sequence. The Pell sequence are defined by the recursive equation:

$$
P_{n}=2 P_{n-1}+P_{n-2}
$$

with initial terms $P_{0}=0$ and $P_{1}=1$. Also, the Pell-Lucas and Modified Pell sequences are defined by the same recurrence but the initial conditions such as $Q_{0}=Q_{1}=2$ and $q_{0}=q_{1}=1$ respectively. It should be noted that there exists an interrelation between the Pell-Lucas and Modified Pell sequences as follows [3]:

$$
Q_{n}=2 q_{n}
$$


Hence, the known properties of the Pell-Lucas numbers can easily be written for the Modified Pell numbers. Thus, a study of Modified Pell numbers involves inevitably familiarity with the Pell-Lucas numbers. The monograph in [4,5] provides many information about these sequences. The following identities can be found in [6-9]:

$$
\begin{gathered}
P_{n}^{2}+P_{n+1} P_{n-1}=\frac{1}{4} Q_{n}^{2} \\
\sum_{k=1}^{n} P_{k}=\frac{P_{n}+P_{n+1}-1}{2} \\
\sum_{k=1}^{n} P_{k}^{2}=\frac{P_{n} P_{n+1}}{2} \\
\sum_{k=1}^{n-1} P_{k} P_{k+1}=\frac{P_{2 n+1}-2 P_{n} P_{n+1}-1}{4}=\frac{P_{2 n-1}+2 P_{n-1} P_{n}-1}{4} \\
\sum_{k=1}^{n} Q_{2 k+1}=\frac{Q_{2 n+2}-6}{2} \\
\sum_{k=1}^{n} Q_{k}^{2}=\frac{Q_{2 n+1}+2(-1)^{n}-4}{2}
\end{gathered}
$$

The Pell, Pell-Lucas and Modified Pell sequences can also be defined with negative subscripts. The terms with the negative subscripts can be obtained to extend these sequences to the left by employing the corresponding recurrence relation. Furthermore,

$$
P_{-n}=(-1)^{n+1} P_{n}, Q_{-n}=(-1)^{n} Q_{n} \text { and } q_{-n}=(-1)^{n} q_{n}
$$

can be found in $[6,7]$.

Let $\left\{t_{n}\right\}_{n=0}^{\infty}$ be a doubly infinite sequence. A Toeplitz matrix is an $n \times n$ matrix such that $T_{n}=\left[t_{i, j}=t_{i-j}: i, j=0,1, \ldots, n-1\right]$. Clearly, the explicit form of this matrix is as follows:

$$
T_{n}=\left[\begin{array}{ccccc}
t_{0} & t_{-1} & t_{-2} & \cdots & t_{-n+1} \\
t_{1} & t_{0} & t_{-1} & \cdots & t_{-n+2} \\
t_{2} & t_{1} & t_{0} & \cdots & t_{-n+3} \\
\vdots & \vdots & \vdots & \ddots & \vdots \\
t_{n-1} & t_{n-2} & t_{n-3} & \cdots & t_{0}
\end{array}\right]
$$

The Frobenius norm and spectral norm of a matrix $A=\left[a_{i j}\right]_{n \times n}$ is defined by; 


$$
\|A\|_{F}=\left(\sum_{i=1}^{n} \sum_{j=1}^{n}\left|a_{i j}\right|^{2}\right)^{\frac{1}{2}} \text { and }\|A\|_{2}=\sqrt{\max _{1 \leq i \leq n}\left|\lambda_{i}\right|}
$$

respectively, where the numbers $\lambda_{i}$ 's are the eigenvalues of the matrix $A^{H} A$, and $A^{H}$ denotes the conjugate of transpose of the matrix $A$. The matrix norms induced by the vector 1 -norm (the largest absolute column sum) and $\infty$-norm (the largest absolute row sums) are as follows:

$$
\|A\|_{1}=\max _{j} \sum_{i}\left|a_{i j}\right| \text { and }\|A\|_{\infty}=\max _{i} \sum_{j}\left|a_{i j}\right|
$$

The maximum column length norm $c_{1}($.$) and the maximum row length norm r_{1}($.$) of an$ matrix of order $n \times n$ are defined as follows:

$$
C_{1}(A)=\max _{j} \sqrt{\sum_{i}\left|a_{i j}\right|^{2}}=\max _{j}\left\|\left[a_{i j}\right]_{i=1}^{m}\right\|_{F} \text { and } r_{1}(A)=\max _{i} \sqrt{\sum_{j}\left|a_{i j}\right|^{2}}=\max _{i}\left\|\left[a_{i j}\right]_{j=1}^{m}\right\|_{F}
$$

Hence, the following inequality holds for any matrix [10,11]:

$$
\frac{1}{\sqrt{n}}\|A\|_{F} \leq\|A\|_{2} \leq r_{1}(B) c_{1}(C)
$$

where $A$ is equal to the Hadamard product of the matrices $B$ and $C$.

In this study, the Toeplitz matrices involving the usual Pell, Pell-Lucas and Modified Pell numbers and their Frobenius, row and column norms are obtained. In addition, the lower and upper bounds of their spectral norms are presented.

\section{Main results}

In this section, the fundamental results are considered. To do this, first of all, the matrices

$$
\begin{aligned}
& \Gamma_{n}=\left[\varphi_{i j}=P_{i+j-1}: i, j=0,1, \ldots, n-1\right]_{n \times n}, \\
& \Lambda_{n}=\left[\kappa_{i j}=Q_{i+j-1}: i, j=0,1, \ldots, n-1\right]_{n \times n},
\end{aligned}
$$

and

$$
\Upsilon_{n}=\left[v_{i j}=q_{i+j-1}: i, j=0,1, \ldots, n-1\right]_{n \times n}
$$

are defined. Note that the entries of the matrices consist of the Pell, Pell-Lucas and Modified Pell numbers. Throughout this paper, the symbol $\xi$ is defined as follows:

$$
\xi=2(-1)^{n}
$$


Hence, the following theorem is given.

Theorem 2.1 Let $\Gamma_{n}$ be an $n \times n$ matrix as in (13). Then,

$$
\left\|\Gamma_{n}\right\|_{F}=\frac{1}{2} \sqrt{P_{n+1}^{2}+P_{n+2} P_{n}-1}=\frac{1}{4} \sqrt{Q_{n+1}^{2}-4}
$$

and

$$
\left\|\Gamma_{n}\right\|_{1}=\left\|\Gamma_{n}\right\|_{\infty}=\frac{P_{n+1}+P_{n}-1}{2}
$$

Proof. Considering the matrix $\Gamma_{n}$ and by the definition of the Frobenius norm, from Eqs. (5$6)$, the following equation can be written:

$$
\begin{aligned}
\left\|\Gamma_{n}\right\|_{F} & =\left(\sum_{i=1}^{n} \sum_{j=1}^{n}\left|\varphi_{i j}\right|^{2}\right)^{\frac{1}{2}}=\left(n P_{0}^{2}+2 \sum_{k=1}^{n-1} \sum_{j=1}^{k} P_{j}^{2}\right)^{\frac{1}{2}}=\left[\sum_{j=1}^{n-1} P_{j} P_{j+1}\right]^{\frac{1}{2}} \\
& =\sqrt{\frac{\left(P_{n+1}+P_{n}+1\right)\left(P_{n+1}+P_{n}-1\right)}{4}}=\sqrt{\frac{P_{n+1}^{2}+P_{n+2} P_{n}-1}{4}}
\end{aligned}
$$

By Eq. (3), the second side of Eq. (17) can be obtained. On the other hand,

$$
\left\|\Gamma_{n}\right\|_{1}=\max _{j} \sum_{i}\left|\varphi_{i j}\right|=\sum_{i=0}^{n-1} P_{i}=\frac{P_{n}+P_{n-1}-1}{2}
$$

and

$$
\left\|\Gamma_{n}\right\|_{\infty}=\max _{i} \sum_{j}\left|\varphi_{i j}\right|=\sum_{i=0}^{n-1} P_{i}=\left\|\Gamma_{n}\right\|_{1}
$$

are directly written from Eq. (4). Thus, the proof is completed.

Now the upper and lower bounds of spectral norm of the matrix $\Gamma_{n}$ are presented.

Theorem 2.2 Let $\Gamma_{n}$ be an $n \times n$ matrix as in (13). Then,

$$
\frac{1}{2} \sqrt{\frac{P_{n+1}^{2}+P_{n+2} P_{n}-1}{n}} \leq\left\|\Gamma_{n}\right\|_{2} \leq \frac{1}{2} \sqrt{P_{n} P_{n-1}\left(2+P_{n-1} P_{n}\right)} .
$$

Proof. By Theorem 1 and from Eq. (12),

$$
\frac{1}{2} \sqrt{\frac{P_{n+1}^{2}+P_{n+2} P_{n}-1}{n}} \leq\left\|\Gamma_{n}\right\|_{2}
$$


is obtained. Also define two matrices such that $U_{n}=\left[u_{i j}\right]_{i, j=1}^{n}$ satisfying $u_{i j}=\left\{\begin{array}{cl}1, & \text { for } j=1 \\ P_{i-j}, & \text { for } j \neq 1\end{array}\right.$ and $V_{n}=\left[V_{i j}\right]_{i, j=1}^{n}$ satisfying $V_{i j}=\left\{\begin{array}{cc}P_{i-j}, & \text { for } j=1 \\ 1, & \text { for } j \neq j\end{array}\right.$, respectively. It can easily be seen that UoV. Thus,

$$
r_{1}(U)=\max _{i} \sqrt{\sum_{j}\left|u_{i j}\right|^{2}}=\sqrt{\sum_{i=n}^{2 n-1}\left|P_{k}\right|^{2}}=\frac{1}{2} \sqrt{\sum_{i=1}^{2 n-1} P_{i}^{2}-\sum_{i=1}^{n-1} P_{i}^{2}}=\sqrt{\frac{P_{2 n} P_{2 n-1}-P_{n} P_{n-1}}{2}}
$$

and

$$
c_{1}(V)=\max _{j} \sqrt{\sum_{i}\left|v_{i j}\right|^{2}}=\sqrt{1+\sum_{i=n}^{2 n-2}\left|P_{k}\right|^{2}}=\sqrt{1+\sum_{i=1}^{2 n-2} P_{i}^{2}-\sum_{i=1}^{n-1} P_{i}^{2}}=\sqrt{1+\frac{P_{2 n-1} P_{2 n-2}-P_{n} P_{n-1}}{2}} .
$$

Considering Eq. (12), the desired result is readily obtained.

Theorem 2.3 Let $\Lambda_{n}$ be an $n \times n$ matrix as in (14). Then, we have

$$
\left\|\Lambda_{n}\right\|_{F}=\sqrt{\frac{Q_{2 n}^{2}-\xi}{2}}
$$

and

$$
\left\|\Lambda_{n}\right\|_{1}=\left\|\Lambda_{n}\right\|_{\infty}=\frac{1}{2}\left(Q_{n}+Q_{n-1}\right)=2 P_{n}
$$

Proof. By the definition of the Frobenius norm,

$$
\begin{aligned}
\left\|\Lambda_{n}\right\|_{F} & =\left(\sum_{i=1}^{n} \sum_{j=1}^{n}\left|\kappa_{i j}\right|^{2}\right)^{\frac{1}{2}}=\left(n Q_{0}^{2}+2 \sum_{k=1}^{n-1} \sum_{j=1}^{k} Q_{j}^{2}\right)^{\frac{1}{2}} \\
& =\left[4 n+\frac{1}{2}\left(Q_{2 n}-6\right)+2 \frac{-1-(-1)^{n}}{2}-4(n-1)\right]^{\frac{1}{2}}=\left[\frac{1}{2} Q_{2 n}-(-1)^{n}\right]^{\frac{1}{2}}
\end{aligned}
$$

can be written, that is, which is desired. Furthermore,

$$
\left\|\Lambda_{n}\right\|_{1}=\max _{j} \sum_{i}\left|\kappa_{i j}\right|=\sum_{i=0}^{n-1} Q_{i}=2+\frac{Q_{n}+Q_{n-1}-4}{2}=\frac{Q_{n}+Q_{n-1}}{2}=P_{n}
$$

and

$$
\left\|\Lambda_{n}\right\|_{\infty}=\max _{i} \sum_{j}\left|\kappa_{i j}\right|=\sum_{i=0}^{n-1} Q_{i}=\left\|\Lambda_{n}\right\|_{1} .
$$


Thus, the proof is completed.

Theorem 2.4 Let $\Lambda_{n}$ be an $n \times n$ matrix as in (14). Then,

$$
\sqrt{\frac{Q_{2 n}-\xi}{2 n}} \leq\left\|\Lambda_{n}\right\|_{2} \leq \frac{1}{4} \sqrt{\left(Q_{2 n-1}-\xi\right)\left(Q_{2 n-1}-\xi+4\right)}
$$

Proof. Considering Theorem 3 and from Eq. (12),

$$
\sqrt{\frac{Q_{2 n}-\xi}{2 n}} \leq\left\|\Lambda_{n}\right\|_{2}
$$

is found, and consider two matrices such that $W_{n}=\left[w_{i j}\right]_{i, j=1}^{n}$ with the entries $W_{i j}=\left\{\begin{array}{cc}1, & \text { for } j=1 \\ Q_{i-j}, & \text { for } i \neq 1\end{array} \quad\right.$ and $Z_{n}=\left[Z_{i j}\right]_{i, j=1}^{n}$ with the entries $Z_{i j}=\left\{\begin{array}{cc}Q_{i-j}, & \text { for } j=1 \\ 1, & \text { for } j \neq 1\end{array}\right.$, respectively. It can easily be seen that $\Lambda_{n}=W o Z$. Thus,

$$
r_{1}(W)=\max _{i} \sqrt{\sum_{j}\left|w_{i j}\right|^{2}}=\sqrt{1+\sum_{i=1}^{n-1}\left|Q_{-i}\right|^{2}}=\frac{1}{2} \sqrt{Q_{2 n-1}-\xi}
$$

and

$$
c_{1}(Z)=\max _{j} \sqrt{\sum_{i}\left|z_{i j}\right|^{2}}=\sqrt{1+\sum_{i=1}^{n-1}\left|Q_{-i}\right|^{2}}=\frac{1}{2} \sqrt{Q_{2 n-1}+4-\xi} .
$$

Considering Equation (12), the proof is readily completed.

Recall that for two matrices $A=\left[a_{i j}\right]_{n \times n}$ and $B=\left[b_{i j}=\alpha a_{i j}\right]_{n \times n}$, we can write

$$
\|A\|=|\alpha|\|B\|
$$

where $\|\bullet\|$ is any matrix norm. Hence, from Eq. (2), the following corollary can be given by using Theorems 3. and 4. without the proof.

Theorem 2.5 Let $\Upsilon_{n}$ be an $n \times n$ matrix as in (15). Then,
i. $\quad\left\|\Upsilon_{n}\right\|=\frac{1}{2 \sqrt{2}} \sqrt{q_{2 n}{ }^{2}-\frac{\varepsilon}{2}}$
ii. $\quad\left\|\Upsilon_{n}\right\|_{1}=\left\|\Upsilon_{n}\right\|_{\infty}=q_{n}+q_{n-1}=P_{n}$ 
iii. $\quad \sqrt{\frac{1}{n}\left(q_{2 n}-\varepsilon\right)} \leq\left\|\Upsilon_{n}\right\|_{2} \leq \frac{1}{2} \sqrt{\left(q_{2 n-1}-\varepsilon\right)\left(q_{2 n-1}-\varepsilon+2\right)}$

where $\varepsilon=\xi / 2$.

\section{References}

[1] Vajda, Steven. Fibonacci and Lucas numbers, and the golden section: theory and applications. (Courier Corporation, 1989.Koshy, Thomas, 2014).

[2] Koshy, Thomasi. Pell and Pell-Lucas numbers with applications. (New York: Springer, 2014).

[3] Horadam, A. F., "Pell identities.” Fibonacci Quarterly 9 (1971): 245-263.

[4] Horadam, A. F., "Unique minimal representation of integers by negatively subscripted Pell numbers." The Fibonacci Quarterly 32 (1994): 202-206.

[5] Horadam, A. F., "Applications of modified Pell numbers to representations." Ulam Quarterly 3(1) (1994): 34-53.

[6] Melham, R., "Sums involving Fibonacci and Pell numbers." Portugaliae Mathematica 56.3 (1999): 309-318.

[7] Kilic, Emrah, and Dursun Tasci, "The linear algebra of the Pell matrix." Bol. Soc. Mat. Mexicana, 11 (2005): 3.

[8] Dasdemir, Ahmet, "On the Pell, Pell-Lucas and modified Pell numbers by matrix method." Applied Mathematical Sciences 5.64 (2011): 3173-3181.

[9] Mathias, Roy, "The spectral norm of a nonnegative matrix." Linear Algebra and its Applications 139 (1990): 269-284. 